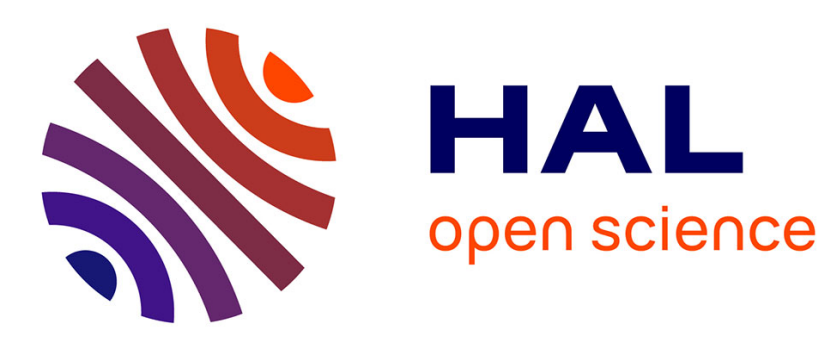

\title{
Solidification of a binary alloy: finite-element, single-domain simulation and new benchmark solutions
}

\author{
Michael Le Bars, M. Grae Worster
}

\section{To cite this version:}

Michael Le Bars, M. Grae Worster. Solidification of a binary alloy: finite-element, single-domain simulation and new benchmark solutions. Journal of Computational Physics, 2006, 216, pp.247-263. 10.1016/j.jcp.2005.12.002 . hal-00084040

\section{HAL Id: hal-00084040 \\ https://hal.science/hal-00084040}

Submitted on 5 Jul 2006

HAL is a multi-disciplinary open access archive for the deposit and dissemination of scientific research documents, whether they are published or not. The documents may come from teaching and research institutions in France or abroad, or from public or private research centers.
L'archive ouverte pluridisciplinaire HAL, est destinée au dépôt et à la diffusion de documents scientifiques de niveau recherche, publiés ou non, émanant des établissements d'enseignement et de recherche français ou étrangers, des laboratoires publics ou privés. 


\title{
Solidification of a binary alloy: finite-element, single-domain simulation and new benchmark solutions
}

\author{
Michael Le Bars* and M. Grae Worster \\ Institute of Theoretical Geophysics, Department of Applied Mathematics and \\ Theoretical Physics, Wilberforce Road, Cambridge, CB3 0WA, United Kingdom
}

\begin{abstract}
A finite-element simulation of binary alloy solidification based on a single-domain formulation is presented and tested. Resolution of phase change is first checked by comparison with the analytical results of Worster [1] for purely diffusive solidification. Fluid dynamical processes without phase change are then tested by comparison with previous numerical studies of thermal convection in a pure fluid [2-4], in a porous medium with a constant porosity $[5,6]$ and in a mixed liquid-porous medium with a spatially variable porosity $[6,7]$. Finally, new benchmark solutions for simultaneous flow through both fluid and porous domains and for convective solidification processes are presented, based on the similarity solutions in corner-flow geometries recently obtained by Le Bars \& Worster [8]. Good agreement is found for all tests, hence validating our physical and numerical methods. More generally, the computations presented here could now be considered as standard and reliable analytical benchmarks for numerical simulations, specifically and independently testing the different processes underlying binary alloy solidification.
\end{abstract}

Key words: solidification of binary alloy, benchmark solutions, finite-element single-domain simulation.

\section{Introduction}

The solidification of multi-component melts occurs in a wide range of industrial processes and natural phenomena, from the casting of high-performance turbine blades to the growth of the inner core of the Earth. It results in the

* Corresponding author. Currently at the IRPHE, UMR 6594, France.

Email address: lebars@irphe.univ-mrs.fr (Michael Le Bars).

$\begin{array}{ll}\text { Preprint submitted to Elsevier Science } & 5 \text { July } 2006\end{array}$ 
formation of a solid with a different composition and in the rejection of a liquid that must be transported away from the interface. This transport is enhanced near protrusions of the front, which therefore grow more rapidly and cause the interface to be unstable: a layer of mixed phase then develops, called a mushy layer. In the mushy layer, the solid forms a porous matrix that continuously evolves because of internal solidification and local dissolution. The flow through this multiphase system is a particularly fascinating and challenging subject for fluid dynamicists, since it involves complex thermal and compositional convective processes in the context of evolving free boundaries (see reviews $[9,10]$ for example).

Solidification processes have been the focus of many investigations. Laboratory experiments have been performed using particular materials, such as aqueous solutions of ammonium chloride [11-13], metallic alloys [14,15], NaCl-water solutions [16] and alcohol-water solutions [9]. From the theoretical point of view, governing equations for the solidifying system have been derived using mixture theory (e.g. [17]), volume-averaging method (e.g. [18]), or by considerations of local thermodynamic balances $[19,20]$. These theoretical studies have sought to identify the key dimensionless parameters controlling solidification. Several numerical investigations have also been performed, which can be divided into two groups. Some models use a single formulation for the three different regions (single-domain approach, see for instance $[21,22]$ ): the system is then resolved on a single mesoscopic scale for the three domains. In other models, the mush, the liquid and the solid are described by independent sets of equations and are resolved separately on their relevant scales (i.e. multiple-domain approach, e.g. $[23,24])$. The major difficulties then consist in the formulation of accurate internal boundary conditions and in following the evolution of the interfaces through time. Until now, the single-domain method has been mostly utilised to determine the various characteristic fields (especially the macrosegregation pattern) at a mesoscopic scale in industrial settings (e.g. [25]), whereas the multiple-domain method has been utilised to study the dynamics of interactions between the different regions in idealized theoretical cases (see for instance the steady-state study [26]). There is yet much to be gained from a systematic and precise study of the full time-dependent convective state in general configurations.

With this in mind, we have developed a single-domain formulation of binary alloy solidification accessible to fine-scale two-dimensional resolution by advanced numerical techniques. Such a formulation eliminates the need for explicit consideration of interface motions and for internal coupling boundary conditions. Hence, we realistically address the interactions between the three different domains and their temporal evolution. In particular, the permeability structure and the topology of the mushy layer are not known in advance: they are calculated simultaneously with solving the coupled equations of mass, momentum, energy and species transport, and the system is entirely free to 
evolve.

In a first paper, our physical single-domain model has been validated by comparisons with results from the multiple-domain formulation in some analytically tractable cases [8]. The present paper has two objectives: we first want to describe and test our numerical simulation; but more generally, we also aim at defining reliable benchmark solutions for numerical models of solidification, based on analytical rather than numerical results. In particular, we want to check specifically the complex thermodynamical processes of binary alloy phase change as well as the intricate motions taking place simultaneously through the fluid melt and the porous mush.

This article is organised as follow. The physical model of binary alloy solidification based on the volume-averaging techniques is briefly outlined in section 2. Section 3 then describes our numerical approach, developed from finiteelement formulations of fluid dynamics problems. The last part of the paper is devoted to various tests of the controllability of our model and its resolution. Section 4 first focuses on a purely diffusive solidification problem, as studied analytically by Worster [1]. In section 5 , we then study classical convective processes without phase change in a pure liquid [2-4], in a fixed porous medium $[5,6]$ and in a mixed porous-liquid domain [6,7]. Finally in section 6 , we present new benchmark solutions for flows through mixed fluid-porous domains and for convective solidification: corner flows in a fluid overlying a porous layer with imposed porosity profiles as well as the complete solidification of a binary alloy in a corner flow are computed and compared with similarity solutions recently published [8].

\section{Physical model}

We consider a binary alloy in a two-dimensional rectangular domain as shown in figure 1 (height $h$ and width $w$ ). Initially, the fluid is stagnant at a given temperature $T_{i}$ and a given concentration $C_{i}$. At time $t=0$, one of the four sides (for instance, in the following, the left side) is suddenly cooled at an imposed temperature $T_{i m p}$ below the eutectic temperature $T_{e}$, whereas the opposite side is maintained at the initial temperature $T_{i}$ and the two other sides remain insulated. A solid phase and a mushy layer then grows from the cold boundary, and convective motions driven by both thermal and solutal buoyancy can occur. Supposing the outer boundaries to be fully rigid and impermeable, velocities and solute flux remain equal to zero on the four sides. 


\subsection{The volume-averaging method}

The transport phenomena in this multiphase solid-liquid system (i.e. transport of mass, momentum, energy, species) can be described in detail by transport equations at the microscopic level characterising the evolution of each phase separately, as well as their interactions. In practice however, and especially from a numerical point of view, this microscopic description is generally not tractable, because of the morphological complexities of the solid-liquid interface and because of the superposition of transport phenomena at various scales. A practical way to examine such problems is thus to work at an intermediate scale, large enough to smooth the morphological complexities, but small enough to capture the global transport properties (i.e. typically several times the pore size of the porous mushy layer). At this mesoscopic scale, transport equations come from the volume-averaging of the microscopic equations over a given control volume. This approach can be compared with the Large-EddySimulation techniques in turbulence (e.g. $[27,28]$ ), in which the motions down to the grid scale are directly computed and motions for the smaller scales are parameterised. Concepts and theorems that are used by the volume-averaging approach are well known and can be found elsewhere (e.g. [18,29]).

\subsection{Transport equations}

The solidifying binary alloy is fully characterised by 5 independent volumeaveraged variables: the enthalpy $H$; the species concentration $C$; the twodimensional Darcy velocity vector $\mathbf{u}$; and the liquid fraction $\epsilon$. Other useful variables can be derived from these primary unknowns, i.e. the temperature $T$, the liquid enthalpy $H_{l}$ and the liquid and solid concentrations $C_{l}$ and $C_{s}$. In the following, we denote the viscosity of the liquid by $\eta$, the density by $\rho_{k}$, the thermal conductivity by $k_{k}$, the heat capacity per unit volume by $c_{p, k}$ and the species diffusivity by $D_{k}$, where $k=l$ for the liquid and $k=s$ for the solid. To keep the problem simple, we suppose that the solid and the liquid have the same density, that the Boussinesq approximation is valid, and that species diffusion in the solid is zero. Besides, we suppose that the permeability is given by the Carman-Kozeny function [30]

$$
\Pi(\epsilon)=\Pi_{0} \frac{\epsilon^{3}}{(1-\epsilon)^{2}},
$$

where $\Pi_{0}$ is a reference permeability. We then define the dimensionless temperature and concentration by

$$
\theta=\frac{T-T_{e}}{T_{i}-T_{e}}, \quad C=\frac{C-C_{e}}{C_{i}-C_{e}},
$$


where $C_{e}$ is the eutectic concentration. We also introduce the characteristic scales for energy $c_{p, l}\left(T_{i}-T_{e}\right)$, length $h$, time $h^{2} / \kappa_{l}$, velocity $\kappa_{l} / h$ and pressure $\rho \kappa_{l}^{2} / h^{2}$, where $\kappa_{l}$ is the thermal diffusivity $\kappa_{l}=k_{l} / c_{p, l}$.

In this context, the dimensionless mesoscopic transport equations can be derived from their microscopic counterpart using the volume-averaging method (see for instance $[18,7,8]$ ). The transport of mass is described by

$$
\nabla \cdot \mathbf{u}=0
$$

The transport of enthalpy is described by

$$
\frac{\partial H}{\partial t}+\mathbf{u} \cdot \nabla H_{l}=\nabla \cdot[\epsilon+(1-\epsilon) k] \nabla \theta
$$

where $k=k_{s} / k_{l}$. Enthalpies and temperature are related through thermodynamical considerations by

$$
\begin{aligned}
& H=H_{s}^{0}+\epsilon L+\left[\epsilon+(1-\epsilon) c_{p}\right]\left[\theta+\frac{T_{e}}{T_{i}-T_{e}}\right], \\
& H_{l}=H_{s}^{0}+L+\theta+\frac{T_{e}}{T_{i}-T_{e}},
\end{aligned}
$$

where $L$ is the latent heat, $H_{s}^{0}$ the reference enthalpy and $c_{p}=c_{p, s} / c_{p, l}$. The transport of species is described by

$$
\frac{\partial C}{\partial t}+\mathbf{u} \cdot \nabla C_{l}=\frac{1}{\mathrm{Le}} \nabla \cdot \epsilon \nabla C_{l}
$$

where Le $=\kappa_{l} / D_{l}$ is the Lewis number. Assuming that the Lever rule applies,

$$
C=\epsilon C_{l}+(1-\epsilon) C_{s}
$$

Finally, the transport of momentum is described by

$$
\begin{aligned}
\frac{\partial}{\partial t} \mathbf{u} & +(\mathbf{u} \cdot \nabla) \frac{\mathbf{u}}{\epsilon}= \\
& -\epsilon \nabla p+\operatorname{Pr} \nabla^{\mathbf{2}} \mathbf{u}+\epsilon \operatorname{Pr}\left(\operatorname{Ra}_{T} \theta-\operatorname{Ra}_{C} C_{l}\right) \mathbf{e}_{\mathbf{y}}-\frac{\operatorname{Pr}}{\operatorname{Da}} \frac{(1-\epsilon)^{2}}{\epsilon^{2}} \mathbf{u}
\end{aligned}
$$

where $p$ is the liquid pressure. The Prandtl number, the thermal and compositional Rayleigh numbers and the Darcy number are respectively defined by 


$$
\begin{aligned}
& \operatorname{Pr}=\frac{\eta}{\rho_{l} \kappa_{l}}, \quad \operatorname{Ra}_{T}=\frac{\alpha \rho_{l} g\left(T_{i}-T_{e}\right) h^{3}}{\kappa_{l} \eta}, \\
& \operatorname{Ra}_{C}=\frac{\beta \rho_{l} g\left(C_{i}-C_{e}\right) h^{3}}{\kappa_{l} \eta}, \quad \mathrm{Da}=\frac{\Pi_{0}}{h^{2}} .
\end{aligned}
$$

\subsection{Closure relationships}

To close the system, we use the simplified phase diagram presented in figure 2 . In dimensionless form, for any given concentration $C$, the liquidus and solidus temperatures are

$$
\begin{aligned}
& \theta_{\text {liquidus }}(C)=\theta_{m}+m\left(C+\frac{C_{e}}{C_{i}-C_{e}}\right), \\
& \theta_{\text {solidus }}(C)=\max \left[0 ; \theta_{m}+\frac{m}{p_{c}}\left(C+\frac{C_{e}}{C_{i}-C_{e}}\right)\right],
\end{aligned}
$$

where $m$ is the dimensionless liquidus slope, $p_{c}$ the partition coefficient and $\theta_{m}$ the dimensionless solidification temperature of the pure component. In terms

of enthalpy (used here as a primary variable), we can calculate the minimum enthalpy of a pure fluid at the concentration $C$

$$
H_{\text {liquidus }}(C)=H_{s}^{0}+L+\theta_{\text {liquidus }}(C)+\frac{T_{e}}{T_{i}-T_{e}},
$$

the maximum enthalpy of a pure solid at the concentration $C$

$$
H_{\text {solidus }}(C)=H_{s}^{0}+c_{p}\left[\theta_{\text {solidus }}(C)+\frac{T_{e}}{T_{i}-T_{e}}\right]
$$

and the enthalpy of a mixed solid-liquid system just above the eutectic, i.e. when $\theta=0, C_{l}=0$ and $C_{s}=\left(p_{c}-1\right) C_{e} /\left(C_{i}-C_{e}\right)$,

$$
H_{\text {eutectic }}(C)=H_{s}^{0}+\epsilon_{e} L+\left[\epsilon_{e}+\left(1-\epsilon_{e}\right) c_{p}\right] \frac{T_{e}}{T_{i}-T_{e}},
$$

where the eutectic liquid fraction $\epsilon_{e}$ is given by (8) as a function of $C$.

Then, for any given $(H, C), 4$ cases are possible. If $H>H_{\text {liquidus }}(C)$, the material is liquid and $\epsilon=1$. If $H<H_{\text {solidus }}(C)$, the material is solid and $\epsilon=0$. If $H_{\text {solidus }}(C) \leq H<H_{\text {eutectic }}(C)$, the material is at the eutectic: $C_{l}=0$, $\theta=0$, and $\epsilon$ is given by (5). If $H_{\text {eutectic }}(C) \leq H \leq H_{\text {liquidus }}(C)$, the system is in a mixed liquid-solid phase strictly above the eutectic: the solid and liquid 
concentration are given by the linear solidus and liquidus relationships (11-12) and $\epsilon$ is the solution in $] 0 ; 1$ ] of the second-order equation obtained from (5) and (8).

\section{Numerical approach}

\subsection{Discretisation in space: finite-element formulation}

We performed the discretisation in space of the governing equations using a finite-element method in a rectangular grid slightly biased towards the walls (see for instance figure 1). Interpolating functions $\Phi_{c}$ are linear in both directions inside each cell $c$. In order to stabilise the advection-diffusion equations, we introduce a Streamline Upwind Petrov-Galerkin (SUPG) term [31]: our test functions are thus of the form

$$
\Phi_{c}+\tau \mathbf{u}^{n} \cdot \nabla \Phi_{c}
$$

The SUPG stabilising parameter $\tau$ is calculated inside each cell by

$$
\tau=\frac{h_{c}}{2\left|\mathbf{u}_{c}\right|}[\operatorname{coth}(\mathrm{Pe})-1 / \mathrm{Pe}], \quad \mathrm{Pe}=\frac{\left|\mathbf{u}_{c}\right| h_{c}}{2 \zeta},
$$

where $\mathbf{u}_{c}$ is the mean velocity in the cell, $h_{c}$ the size of the cell in the direction

of $\mathbf{u}_{c}, \zeta$ the dimensionless diffusion coefficient, respectively equal to $1, \epsilon_{c} / \mathrm{Le}$ and $\epsilon_{c} \operatorname{Pr}$ for the transport of energy, species and momentum, and where $\epsilon_{c}$ is the mean liquid fraction in the cell.

\subsection{Discretisation in time and solution strategy}

Our initial state at $t=0$ corresponds to a pure fluid at temperature $\theta=1$ everywhere except - for instance - on the left side, where a pure solid at temperature $\theta_{i m p}$ appears instantaneously. Hence, in terms of primary variables, initial conditions are $\mathbf{u}=\mathbf{0}, C=1, \epsilon=1$ except $\epsilon_{x=0}=0, H=$ $H_{s}^{0}+L+\left[1+T_{e} /\left(T_{i}-T_{e}\right)\right]$ except $H_{x=0}=H_{s}^{0}+c_{p}\left[\theta_{i m p}+T_{e} /\left(T_{i}-T_{e}\right)\right]$. We then compute the hydrostatic pressure $p$.

Knowing all fields at time $t^{n}$, we successively compute the various fields at time $t^{n+1}=t^{n}+\Delta t$ using the following strategy and time discretisation. First, 
$H^{n+1}$ and $C^{n+1}$ are computed implicitly from (4) and (7), using the following approximations for the liquid enthalpy and the liquid concentration $[32,7]$ :

$$
\begin{aligned}
& H_{l}^{n+1}=H_{l}^{n}+a^{n}\left(H^{n+1}-H^{n}\right) \text { where } a^{n}=\left[\frac{d H_{l}}{d H}\right]^{n}, \\
& C_{l}^{n+1}=C_{l}^{n}+\left(C^{n+1}-C^{n}\right) .
\end{aligned}
$$

Then, $\epsilon^{n+1}$ is computed from $\left(C^{n+1}, H^{n+1}\right)$, using the phase diagram closure relationships describe in $\S 2.3$. We also compute the secondary fields $\theta^{n+1}, H_{l}^{n+1}$ and $C_{l}^{n+1}$. Finally, the Darcy-Brinkman equation is solved using the fractional step method $[33,34]$. We write the velocity and pressure fields in the form

$$
\mathbf{u}^{n+1}=\mathbf{u}^{*}+\Delta \mathbf{u}, \quad p^{n+1}=p^{*}+\Delta p
$$

and split the Darcy-Brinkman equation (9) in two approximated parts:

$$
\begin{aligned}
& \frac{\mathbf{u}^{*}-\mathbf{u}^{n}}{\Delta t}+\left(\mathbf{u}^{n} \cdot \nabla\right) \frac{\mathbf{u}^{*}}{\epsilon^{n+1}}=-\epsilon^{n+1} \nabla p^{*}+\operatorname{Pr} \nabla^{\mathbf{2}} \mathbf{u}^{*}+ \\
& \epsilon^{n+1} \operatorname{Pr}\left(\operatorname{Ra}_{T} \theta^{n+1}-\operatorname{Ra}_{C} C_{l}^{n+1}\right) \mathbf{e}_{\mathbf{y}}-\frac{\operatorname{Pr}}{\operatorname{Da}} \frac{\left(1-\epsilon^{n+1}\right)^{2}}{\left(\epsilon^{n+1}\right)^{2}} \mathbf{u}^{*} \\
& \frac{\Delta \mathbf{u}}{\Delta t}=-\epsilon^{n+1} \nabla \Delta p
\end{aligned}
$$

From (3), the divergence of (22) gives the Poisson equation

$$
\frac{\nabla \cdot \mathbf{u}^{*}}{\Delta t}=\nabla \cdot \epsilon^{n+1} \nabla \Delta p
$$

We then solve the Darcy-Brinkman equation in successive cycles of 3 steps. We first write $p^{*}=p^{n}$. We then compute the fictitious velocity field $\mathbf{u}^{*}$ from (21), with the boundary conditions $\mathbf{u}^{*}=\mathbf{0}$ on the walls. We then compute the correction in pressure from (23), with the boundary conditions $\Delta p(0,0)=0$ and $\nabla \Delta p \cdot \mathbf{n}=0$ on the sides. We finally replace $p^{*}$ by $p^{*}+\Delta p$ in $(21)$ and repeat until convergence, i.e. until changes in $\mathbf{u}^{*}$ between two successive cycles are sufficiently small:

$$
\max \left(\frac{\left|\mathbf{u}^{*}-\mathbf{u}^{* *}\right|}{\max \left(\left|\mathbf{u}^{*}\right|\right)}\right)<\text { Tol, }
$$

where $\mathbf{u}^{* *}$ is the velocity field determined at the previous cycle and Tol is a fixed value, chosen in agreement with the expected precision of the code (this will be discussed in the following). 
No simplification regarding the variations of $\epsilon$ is introduced in the resolution of the energy and species transport equations. However, for simplicity and to avoid extra-sensitivity on $\epsilon$ in the resolution of the Darcy-Brinkman equation, the mean value $\epsilon_{m}$ of $\epsilon$ inside each cell is used when solving (21) (see also [7]). Besides, to avoid singularity, $\epsilon_{m}$ is bounded by a small value $\epsilon_{\min }$ when it goes to 0: the Darcy term in (21) then ensures that $\mathbf{u}^{*} \rightarrow 0$, as expected in the solid. As a test, instead of computing $H^{n+1}, C^{n+1}, \epsilon^{n+1}$ and $\mathbf{u}^{n+1}$ directly, we compute intermediate fields $\hat{H}, \hat{C}, \hat{\epsilon}$, and $\hat{\mathbf{u}}$ and repeate the whole scheme until convergence is obtained between all fields. This process, which increases the necessary computation time, does not significantly affect the results, provided the time-step is not too large. So we did not adopt it generally.

It is worth noticing here that our code uses a first-order approach in time, linear interpolation functions in space and approximations for the liquid concentration and enthalpy (see (18) and (19)). Hence, we can not expect a huge accuracy. With the standard $81 \times 81$ grid, a typical precision around one percent seems reasonable. This will be confirm in the following by the comparisons between our numerical results and the analytical results available in some interesting cases (see $\S 4$ and $\S 6$ ). Accordingly, in the following computations, the numerical parameters Tol and $\epsilon_{\min }$ are taken equal to $1 \%$ and all results are given with three significative figures. Computations performed with a smaller value $0.1 \%$ do not show significant differences.

To control the ability of our physical and numerical approaches to simulate fluid flows as well as thermodynamical processes of solidification correctly, series of tests have been performed and are presented in the following.

\section{Diffusive solidification}

Our first validation test focuses on thermodynamic processes of phase change. To do so, we eliminate all motions in the tank by setting $\operatorname{Ra}_{T}=\operatorname{Ra}_{C}=0$, and we study purely diffusive solidification, for which analytical solutions have been determined using a multiple-domain approach [1].

In the general configuration described previously, we first verify that in the absence of fluid motions, no variables depend on depth $y$, but only on time $t$ and width $x$. We then adapt our code to the configuration of Worster [1]. Since analytical results are obtained in a semi-infinite domain, we impose a very large aspect ratio $w / h=200$ and choose a $242 \times 4$ grid, largely refined towards the cold wall. Besides, the imposed boundary temperature $T_{i m p}$ in [1] remains above the eutectic. In order to induce the formation of a pure solid in our simulation with a partition coefficient $p_{c}=0$, we slightly change the boundary conditions. We suppose that a solid crystal $(\epsilon=0)$ instantaneously 
forms on the left wall $x=0$ at $t=0$. According to the phase diagram, its solid concentration is $C_{s}=0$ in dimensional form. Hence, in terms of primary dimensionless variables $(H, C)$, initial conditions on the left side are

$$
H_{i m p}=H_{s}^{0}+c_{p}\left[\theta_{i m p}+\frac{T_{e}}{T_{i}-T_{e}}\right], \quad C_{i m p}=\frac{-C_{e}}{C_{i}-C_{e}} .
$$

Since we neglect diffusion in the solid, we impose the concentration (and the enthalpy) to remain equal to this value at the boundary.

According to [1], dimensionless positions of the solid/mush interface and the mush/liquid interface in an infinite domain are respectively

$$
a(t)=2 \lambda_{a} \sqrt{\frac{t}{\mathrm{Le}}}, \quad b(t)=2 \lambda_{b} \sqrt{\frac{t}{\mathrm{Le}}},
$$

where $\lambda_{a, b}$ are constants depending on the physical parameters of the system and on the boundary conditions. Using our numerical simulation, we determine the temporal evolution of the solid/mush interface (i.e. contour $\epsilon=0$ ) and the mush/liquid interface (i.e. contour $\epsilon=1$ ) and normalise them by $2 \sqrt{t / \text { Le }}$. An example is presented in figure 3 . Because of the discretisation in space, the curve exhibits sawtooth shape, with sharp increases corresponding to the displacement of the interface from one grid point to the next one and continuous decrease corresponding to the interface remaining at the same grid point while time increases. Within the uncertainty introduced by this phenomenom (i.e. $4.5 \%$ in this case), one can notice that the mush-liquid and the mush-solid interface positions converge towards constant values, to which they remain equal until the effects of the finite domain are felt. Values of these constants $\lambda_{a, b}$ are presented in figure 4 as a function of $T_{\text {liquidus }}\left(C_{i}\right)-T_{\text {imp }}$, in comparison with [1]. Analytical and numerical results agree well within the precision of the numerics, hence validating both our physical and numerical approaches of thermodynamical processes.

For large driving temperature differences, the analytical values for the mushliquid interface are closer to the lower bound of the numerical values (determined just before the interface jumps to the next grid point). This agrees with the fact that the contour $\epsilon=1$ overestimates the location of the interface (i.e. the interface is somewhere between the first point where $\epsilon=1$ and the one just before). We similarly explain why the results for the mush-solid interface always seem to underestimate the analytical results. Nevertheless, since we use linear approximations inside each cell, we decided for coherence to take the mean value of the contours $\epsilon=1$ and $\epsilon=0$ as numerical results. One can also notice that for $T_{\text {liquidus }}\left(C_{i}\right)-T_{i m p} \leq 1.99$, the analytical study predicts a liquid-solid interface without mush. The numerical results still exhibit the 
relevant behaviour, but since our code is not designed for dealing with such a sharp change in the liquid fraction, quantitative results do not agree as well as for $T_{\text {liquidus }}\left(C_{i}\right)-T_{i m p}>1.99$.

To the best of our knowledge, the results presented here correspond to the first direct comparison between analytical and computational solutions of diffusive solidification. We thus suggest that these computations should now be considered as standard and simple benchmarks of the controllability and resolution of binary alloy simulations.

\section{Convection tests}

We now focus on fluid flows without phase change and provide some classical tests regarding convective processes in a fluid, in a porous domain with constant porosity and in a mixed non-reacting porous-liquid domain.

\subsection{Thermal convection in a pure fluid}

We consider a pure fluid in a square cavity, the imposed temperature on the left side being above the liquidus temperature $\left(\theta_{i m p}=2\right)$. Thermal convective patterns then develop in the tank, while the bulk concentration and the liquid fraction remain equal to 1 . Computations are pursued until a steady state is reached, i.e. until

$$
\max \left(\left|\theta^{n+1}-\theta^{n}\right|\right)<10^{-3}
$$

We then measure the maximum vertical velocity at the mid-height, the maximum horizontal velocity at the mid-width and the maximum, minimum and average values over the left and right sides of the Nusselt number $\mathrm{Nu}$ (i.e. the dimensionless heat flux), defined by

$$
\mathrm{Nu}=\frac{\partial \theta}{\partial x}
$$

We verify that the heat fluxes at the two sides are equal in steady state.

First, several computations were performed at fixed Rayleigh and Prandtl numbers $\mathrm{Ra}=10^{6}, \operatorname{Pr}=0.71$ in order to test and assess grid independence of the solution scheme (see table 1 ). The $81 \times 81$ biased grid shown in figure 1 is found to be adequate for all the simulations within the precision of the code, 
also keeping the computation times within acceptable duration from minutes to hours. This grid is thus systematically used in the following computations.

A parametric study has also been performed by systematically changing the Rayleigh number. Results are presented in table 2 for a Prandtl number $\mathrm{Pr}=$ 0.71, in comparison with previous studies. Good agreement is found over a very large parameter range $10^{3} \leq \mathrm{Ra} \leq 10^{8}$. The maximum variation of our results compared to the mean values over all simulations is about $3.2 \%$, and our computations always remain within the typical standard deviations of previous ones. This validates our numerical resolution of the (pure fluid) Navier-Stokes and temperature equations.

\subsection{Thermal convection in a fixed porous medium}

We now consider the same situation but in a fixed porous medium with a constant porosity $\epsilon_{0}$. We suppose that the thermal conductivity and the heat capacity of the solid and the fluid are equal (i.e. $k=1$ and $c_{p}=1$ ). For comparison with previous studies, we define here a modified Darcy number

$$
\overline{\mathrm{Da}}=\mathrm{Da} \frac{\epsilon_{0}^{3}}{\left(1-\epsilon_{0}\right)^{2}} .
$$

Computations are pursued until a steady state is reached, and the average value over the left and right sides of the Nusselt number (equal in steady state) is measured. We performed three series of calculations. First, following [6], the advective terms $(\mathbf{u} \cdot \nabla) \mathbf{u} / \epsilon_{0}$ of the Darcy-Brinkman equation $(9)$ are neglected and the effects of porosity are removed (i.e. we take $\epsilon_{0}=1$ with a finite value of $\overline{\mathrm{Da}}$ ). The average value of $\mathrm{Nu}$ is then independent of the Prandtl number, and only depends on the Rayleigh and modified Darcy numbers. Results are presented in table 3 , and agree within a few percent with previous studies. This validates our numerical treatment of the linear drag term in the DarcyBrinkman equation.

We then performed computations taking the advective and porosity terms into account, with a Prandtl number $\mathrm{Pr}=1$ and a liquid fraction respectively equal to 0.4 and 0.9 . As shown in table 4 , the effects of advective terms and $\epsilon_{0}$ in our model are qualitatively the same as in [6]: in comparison with table 3 , lower heat flux is generally found when inertial effects are included and when $\epsilon_{0}$ decreases, the variations being larger at large Rayleigh and Darcy numbers. However, quantitative results rather disagree: we predict a larger sensitivity, especially at relatively large Darcy number (see results for $\overline{\mathrm{Da}}=10^{-2}$ in table 4). This comes from the fact that Nithiarasu et al. [6] also include in their 
computation a non-linear drag term

$$
-\frac{1.75}{\sqrt{150}} \sqrt{\frac{u^{2}+v^{2}}{\epsilon_{0} \overline{\mathrm{Da}}}} \mathbf{u}
$$

which is not used here. This term should be compared with the linear drag term $\epsilon_{0}(\operatorname{Pr} / \overline{\mathrm{Da}}) \mathbf{u}$. In the context of motions in a fixed porous medium, the nonlinear drag term is important either at large modified Darcy number $\overline{\mathrm{Da}}$ and/or at small imposed liquid fraction $\epsilon_{0}$ and/or at large velocity (i.e. large Rayleigh number). This agrees with the results presented in table 3. Nevertheless, using the scaling of solidification problems, the ratio between the non-linear and linear drag terms is proportional to $\sqrt{\mathrm{Da}} /(1-\epsilon)^{2}$ : provided the Darcy number is very small (which is usually the case in solidification), non-linear terms could only play a role around the liquid-mush interface $\epsilon=1$, where all drag terms rapidly disappear, the flow being controlled by the standard fluid advective and viscous terms. That is why the non-linear drag is not included in the present simulation (see also for instance $[18,32,7]$ ).

\subsection{Thermal convection in a mixed fluid-porous medium}

Following [6,7], we also studied thermal convection in a square cavity with a fixed but spatially-variable porosity, linearly varying from 0.4 at the walls to 1 at a dimensionless distance larger than 0.3 (figure $5 a$ ). Our purpose here is to test the ability of the numerics to describe simultaneous motions through a porous medium and a pure liquid. Computations are pursued until a steady state is reached, and streamlines are presented in figure 5. As expected, the main motions correspond to a convective cell mostly focused into the fluid part of the system. Besides, for left/right imposed boundary temperatures $\theta=2.0 / 1.0$, we observe a twin counter-rotating vortex in the porous medium near the hot wall (figure $5 b$ ): this agrees with results of Nithiarasu et al. [6] and Zabaras \& Samanta [7].

However, one may wonder why the original symmetry of the system is broken in the steady-state solution. Indeed, the only source of symmetry breaking comes from the imposed boundary conditions $\theta=2.0 / 1.0$. To further investigate this problem, we thus performed the same study with imposed boundary temperatures $\theta=-1.0 /-2.0$ and for fully symmetrical boundary conditions $\theta=0.5 /-0.5$. The first configuration then leads to a counter-rotating vortex near the cold wall (figure $5 c$ ), whereas in the second configuration, the twin vortices totally disappear (figure $5 d$ ). All these results remain identical when starting from a linear temperature profile, from an isothermal fluid with a sharp change at the hot wall or from an isothermal fluid with a sharp change at the cold wall. 
From a physical point of view, such changes in boundary temperatures alone can not be responsible for changes in steady-state patterns. One may thus wonder whether these three flows actually derive from a physical symmetrybreaking bifurcation. However, starting from a steady state obtained from the boundary conditions $\theta=2.0 / 1.0$ and suddendly changing them by $\theta=$ $-1.0 /-2.0$, it turns out to be impossible to maintain the counter-rotating vortex near the hot wall: instead, it rapidly disappears and a new vortex forms near the cold wall, demonstrating that each of the three states is linked with particular boundary conditions. Hence, we conclude that the suprising double-vortex pattern is likely to be due to numerical rather than physical effects.

Explaining the precise numerical origin of this phenomenom is beyond the scope of this paper. We expect that it is due to the initial determination of the hydrostatic pressure, which strongly depends on the effective values of the imposed temperatures. In the configuration studied here (i.e. horizontal convection), the system is always unstable and the hydrostatic pressure is not physically relevant; nevertheless, to deal with the initial conditions (i.e. $\mathbf{u}=\mathbf{0}$ whereas the temperature profile is already imposed), it is always computed numerically and has a long-term influence on the convective patterns.

Regarding the present work, we just want to highlight here that the doublevortex pattern should not be considered as a relevant validation test for simultaneous flows through a porous medium and a pure liquid. Instead, we define in the following new benchmarks based on analytical solutions of corner flows [8].

\section{Corner flow tests}

In order to test our code for simultaneous flow through porous and fluid domains as well as for convective solidification processes, we have developed a series of benchmark solutions with various configurations in a corner-flow geometry. To the best of our knowledge, the following tests correspond to the first simple and reliable analytical tests for numerical simulations of binary alloy solidification.

\subsection{Corner flow in a fluid overlying a porous layer with an imposed porosity}

We consider a corner flow in a domain $0 \leq y \leq 1,0 \leq x \leq w / h$, with pure fluid in the upper half and a porous matrix saturated with the same fluid in the lower half (see figure 6). At $y=1$, a purely vertical input velocity 
$\mathbf{u}=\left(0,-V_{\text {imp }}\right)$ is imposed, whereas no vertical velocity $v=0$ and no horizontal shear $\partial u / \partial y=0$ are imposed at the lower wall. Boundary conditions at the left wall are $u(x=0, y)=0$ and $\partial v / \partial x(x=0, y)=0$. We further suppose that the porosity depends on the vertical position $y$ only and that the flow is sufficiently slow to neglect inertial effects.

As shown in [8], similarity solutions for the steady-state velocity vector in the limit $w / h \rightarrow \infty$ can be found in the form

$$
\frac{\mathbf{u}}{V_{i m p}}=\left[-x f^{\prime}(y), f(y)\right]
$$

where $f$ is a function of the depth $y$ only. Our purpose here is to use these similarity solutions for various porosity profiles as benchmark tests for our numerics. To do so, we choose in our code a large aspect ratio $w / h=10$ and impose a purely horizontal constant velocity at the right wall $\mathbf{u}=\left(+V_{i m p} \times w / h, 0\right)$, which ensures overall mass conservation in the domain. These downstream boundary conditions of course disagree with the similarity solution (31) obtained for an infinite domain. Nevertheless, as shown in figure 6, their influence on the global solution is only felt over a limited distance from the right wall, typically comparable to the height of the domain.

Two configurations presented in [8] are tested here: (i) with a porosity changing linearly from 1 at the interface $y=1 / 2$ to a fixed value $\epsilon_{0}$ at $y=0$ and (ii) with a constant porosity $\epsilon_{0}$ through the whole lower half domain. In both cases, starting from $\mathbf{u}=\mathbf{0}$ everywhere except at the top and right boundaries, a steady state is rapidly reached that exhibits good agreement with the similarity profiles (figure 7 ). The maximum relative error defined by

$$
\max \left(\frac{\mid \text { theoretical value }- \text { numerical value } \mid}{\max (\mid \text { theoretical value } \mid)}\right)
$$

is equal to $0.8 \%$ and is obtained in the second case, where the code has to deal with the sharp jump of porosity and permeability at the interface. These configurations thus provide rigorous tests for flows through mixed porous-fluid domains.

\subsection{Directional solidification into a corner flow}

We now consider a binary alloy in the corner-flow geometry with the boundary conditions introduced in the previous section, and we further suppose that the incoming fluid at $y=1$ has a fixed temperature $T_{i}$ and a fixed concentration $C_{i}$, while the temperature of the lower boundary at $y=0$ is fixed 
just above the eutectic temperature $T_{e}$. A mushy layer then grows from this lower boundary, leading to a time-evolving porosity field. Following classical studies in solidification, we suppose that the whole system is pulled vertically downward at a constant rate $V_{\text {pull }}$ and we look for a stationary solution in the moving frame of reference. This means that additional terms $-V_{\text {pull }} \partial H / \partial y$ and $-V_{\text {pull }} \partial C / \partial y$ must be introduced on the left side of equations (4) and (7) respectively.

In the limiting case $w / h \rightarrow \infty$ and Le $\rightarrow \infty$, Le Bars \& Worster [8] found a steady-state similarity solution, where the velocity field has the form (31) and all other fields (i.e. temperature, bulk concentration, liquid fraction, enthalpy) depend on the depth $y$ only. In order to test our numerical simulation, we thus attempt to reproduce these results in choosing a large aspect ratio $w / h=10$ and a large Lewis number Le $=1000$. Boundary conditions for the enthalpy and bulk concentration are $\partial H / \partial x=0$ and $\partial C / \partial x=0$ on the left and right walls, and $\partial C / \partial y=0$ at the bottom. Results are shown in figure 8 . As previously, because of the finite aspect ratio, the situation is not exactly identical to the similarity solution, but the effects remain mostly limited to the right-most part of the domain (typically $9 \leq x \leq 10$ ). All the other results agree with the similarity solution. The relative error for all field remains within $1 \%$, even for the bulk concentration where one could expect the finite Lewis number and the artificial boundary conditions on the right wall to play an important role. We thus conclude that this corner-flow configuration is particularly adapted to test solidification codes, which aim at determining realistic macrosegregation patterns of binary alloy solidification in industrial or natural settings.

\section{Conclusion}

In this paper, a finite-element simulation of binary alloy solidification based on a single-domain physical approach is presented. The ability of the code to correctly simulate both fluid dynamical and thermodynamical processes is carefully checked with previous works. In particular, we present comparisons between analytical and numerical results for purely diffusive solidication, for simultaneous flow through both fluid and porous domains in a corner flow, and for convective solidification into a corner flow, based on similarity solutions determined by Worster [1] and Le Bars \& Worster [8]. These various configurations allow us to test specifically and independently the different processes underlying binary alloy solidification, and could now be considered as standard and simple benchmark solutions for the validation of numerical simulations.

As far as our numerical tool is concerned, good agreement is found for all tests, hence validating the physical and numerical approaches. We now plan 
to use this code to study the various convective regimes within the mushy layer during binary alloy solidification. In particular, we will systematically describe the characteristics of dissolution channels (chimneys) that appear in various natural (sea ice, Earth's core,...) and industrial (casting industry) systems (see for instance [10]). In addition to experimental results, our numerical approach will help to define general scaling laws and to understand the large-scale consequences of these small-scale processes.

The authors would like to acknowledge support from the Leverhulme Trust and from the European Community (Marie Curie Intra European Fellowship FP6501040). We are also grateful to L. Duchemin for several helpful discussions and for examining an earlier draft of this manuscript.

\section{References}

[1] M. G. Worster, Solidification of an alloy from a cooled boundary, J. Fluid Mech. 167 (1986) 481-501.

[2] G. de Vahl Davis, Natural convection of air in a square cavity: a bench mark numerical solution, Int. J. Num. Meth. in Fluids 3 (1983) 249-264.

[3] D. A. Mayne, A. S. Usmani, M. Crapper, h-adaptive finite element solution of high rayleigh number thermally driven cavity problem, Int. J. Numer. Meth. Heat Fluid Flow 10 (2000) 598-615.

[4] D. C. Wan, B. S. V. Patnaik, G. W. Wei, A new benchmark quality solution for the buoyancy driven cavity by discrete singular convolution, Numer. Heat Transfer 40 (2001) 199-228.

[5] G. Lauriat, V. Prasad, Non-darcian effects on natural convection in a vertical porous enclosure, Int. J. Heat Mass Transf 32 (1989) 2135-2148.

[6] P. Nithiarasu, K. N. Seetharamu, T. Sundararajan, Natural convective heat transfer in an enclosure filled with fluid saturated variable porosity medium, Int. J. Heat Mass Transf 40 (1997) 3955-3967.

[7] N. Zabaras, D. Samanta, A stabilized volume-averaging finite element method for flow in porous media and binary alloy solidification processes, Int. J. Numer. Meth. Engng 60 (2004) 1103-1138.

[8] M. Le Bars, M. G. Worster, Interfacial conditions between a pure fluid and a porous medium: implications for binary alloy solidification, J. Fluid Mech., in press.

[9] M. G. Worster, Convection in mushy layers, Ann. Rev. Fluid Mech. 29 (1997) 91-122. 
[10] M. G. Worster, Solidification of fluids, in: G. K. Batchelor, H. K. Moffat, M. G. Worster (Eds.), Perspectives in Fluid Dynamics, Cambridge University Press, 2000, pp. 393-446.

[11] S. M. Copley, A. F. Giamei, S. M. Johnson, M. F. Hornbecker, The origin of freckles in unidirectionally solidified castings, Metall. Trans. 1 (1970) 2193-2204.

[12] S. Tait, C. Jaupart, Compositional convection in a reactive crystalline mush and melt differentiation, J. Geophys. Res. 97(B5) (1992) 6735-6759.

[13] C. F. Chen, Experimental study of convection in a mushy layer during directional solidification, J. Fluid Mech. 293 (1995) 81-98.

[14] A. Hellawell, J. R. Sarazin, R. S. Steube, Channel convection in partly solidified systems, Phil. Trans. R. Soc. Lond. 345 (1993) 507-544.

[15] M. I. Bergman, D. R. Fearn, J. Bloxham, M. C. Shannon, Convection and channel formation in solidifying pb-sn alloys, Metall. Mater. Trans. A 28 (1997) 859-866.

[16] J. S. Wettlaufer, M. G. Worster, H. E. Huppert, Natural convection during solidification of an alloy from above with application to the evolution of sea ice, J. Fluid Mech. 344 (1997) 291-316.

[17] R. N. Hills, D. E. Loper, P. H. Roberts, A thermodynamically consistent model of a mushy zone, Q. J. Mech. Appl. Math. 36 (1983) 505-539.

[18] C. Beckermann, R. Viskanta, Natural convection solid/liquid phase change in porous media, Int. J. Heat Mass Transfer 31 (1988) 35-46.

[19] A. C. Fowler, The formation of freckles in binary alloys, IMA J. Appl. Maths. 35 (1985) 159-174.

[20] M. G. Worster, The dynamics of mushy layers, in: S. H. Davis, H. E. Huppert, U. Müller, M. G. Worster (Eds.), Interactive Dynamics of Convection and Solidification, Kluwer, 1992, pp. 113-138.

[21] S. D. Felicelli, J. C. Heinrich, D. R. Poirier, Simulation of freckles during vertical solidification of binary alloys, Metall. Trans. B 22 (1991) 847-859.

[22] M. C. Schneider, J. P. Gu, C. Beckermann, W. J. Boettinger, U. R. Kattner, Modeling of micro- and macrosegregation and freckle formation in single-crystal nickel-base superalloy directional solidification, Metall. Mater. Trans. A 28 (1997) 1517-1531.

[23] T. P. Schulze, M. G. Worster, Weak convection, liquid inclusions and the formation of chimneys in mushy layers, J. Fluid Mech. 388 (1999) 197-215.

[24] T. P. Schulze, M. G. Worster, A time-dependent formulation of the mushy zone free boundary problem, J. Fluid Mech., in press.

[25] J. Gu, C. Beckermann, Simulation of convection and macrosegregation in a large steel ingot, Met. and Mat. Trans. A 30A (1999) 1357-1366. 
[26] C. A. Chung, M. G. Worster, Steady-state chimneys in a mushy layer, J. Fluid Mech. 455 (2002) 387-411.

[27] M. Lesieur, Numerical simulations of turbulence in shear flows, in: F. T. M. Nieuwstadt (Ed.), Applied Scientific Research, Advances in turbulence IV, Kluwer, 1993, pp. 345-351.

[28] P. Moin, K. Squires, W. Cabot, S. Lee, A dynamic subgrid-scale model for compressible turbulence and scalar transport, Phys. Fluids A3 (1991) 27462757 .

[29] S. Whitaker, The method of volume averaging, Kluwer Academic Publishers, Dordrecht, The Netherlands, 1999.

[30] J. Bear, Dynamics of Fluids in Porous Media, Dover Publications, 1972.

[31] A. Brooks, T. Hughes, Streamline upwind/petrov-galerkin formulations for convection dominated flows with particular emphasis on the incompressible navier-stokes equations, Comp. Meth. in Appl. Mech. Eng. 32 (1982) 199-259.

[32] T. U. Kaempfer, M. Rappaz, Modelling of macrosegregation during solidification processes using an adaptive domain decomposition method, Modelling Simul. Mater. Sci. Eng. 11 (2003) 575-597.

[33] A. J. Chorin, Numerical solution of the navier-stokes equations, Math. Comp. 22 (1968) 745-762.

[34] S. V. Patankar, Numerical Heat Transfer and Fluid Flow, Hemisphere, New York, 1980. 
Table 1

Maximum vertical velocity at the mid-height, maximum horizontal velocity at the mid-width and maximum, minimum and average Nusselt numbers computed with different grids for the thermal convection in a pure fluid $\left(\mathrm{Ra}=10^{6}, \operatorname{Pr}=0.71\right)$.

\begin{tabular}{c|c|c|c|c|c} 
grid & $\max \left(v_{y=0.5}\right)$ & $\max \left(u_{x=0.5}\right)$ & $\max (\mathrm{Nu})$ & $\min (\mathrm{Nu})$ & $\mathrm{av.}(\mathrm{Nu})$ \\
\hline $21 \times 21$ & 223 & 63.4 & 21.2 & 0.903 & 8.84 \\
$41 \times 41$ & 222 & 65.2 & 17.9 & 0.958 & 8.87 \\
$61 \times 61$ & 221 & 65.0 & 17.5 & 0.968 & 8.83 \\
$81 \times 81$ & 220 & 64.7 & 17.5 & 0.972 & 8.82 \\
$101 \times 101$ & 220 & 64.7 & 17.6 & 0.973 & 8.83
\end{tabular}


Table 2

Comparison of the maximum vertical velocity at the mid-height, of the maximum horizontal velocity at the mid-width and of the maximum, minimum and average Nusselt numbers as a function of the Rayleigh number for the thermal convection in a pure fluid $(\mathrm{Pr}=0.71)$.

\begin{tabular}{c|c|c|c|c|c|c}
$\mathrm{Ra}_{T}$ & & $\max \left(v_{y=0.5}\right)$ & $\max \left(u_{x=0.5}\right)$ & $\max (\mathrm{Nu})$ & $\min (\mathrm{Nu})$ & av. $(\mathrm{Nu})$ \\
\hline $10^{3}$ & this study & 3.70 & 3.65 & 1.51 & 0.691 & 1.12 \\
& {$[2]$} & 3.679 & 3.634 & 1.50 & 0.692 & 1.12 \\
& {$[3]$} & 3.6962 & 3.6493 & 1.5062 & 0.6913 & \\
& {$[4]$} & 3.686 & 3.489 & 1.501 & 0.691 & 1.117 \\
\hline \multirow{2}{*}{$10^{4}$} & this study & 19.6 & 16.2 & 3.53 & 0.585 & 2.24 \\
& {$[2]$} & 19.51 & 16.2 & 3.53 & 0.586 & 2.243 \\
& {$[3]$} & 19.6177 & 16.1798 & 3.5305 & 0.5850 & \\
& {$[4]$} & 19.79 & 16.122 & 3.579 & 0.577 & 2.254 \\
\hline \multirow{2}{*}{$10^{5}$} & this study & 68.7 & 34.8 & 7.72 & 0.726 & 4.52 \\
& {$[2]$} & 68.22 & 34.81 & 7.71 & 0.729 & 4.52 \\
& {$[3]$} & 68.6920 & 34.7741 & 7.7084 & 0.7282 & \\
& {$[4]$} & 70.63 & 33.39 & 7.945 & 0.698 & 4.598
\end{tabular}


Table 2 (continue)

\begin{tabular}{c|c|c|c|c|c|c}
$\mathrm{Ra}_{T}$ & & $\max \left(v_{y=0.5}\right)$ & $\max \left(u_{x=0.5}\right)$ & $\max (\mathrm{Nu})$ & $\min (\mathrm{Nu})$ & av. $(\mathrm{Nu})$ \\
\hline \multirow{2}{*}{$10^{6}$} & this study & 220 & 64.7 & 17.5 & 0.972 & 8.82 \\
& {$[2]$} & 216.75 & 65.33 & 17.92 & 0.989 & 8.8 \\
& {$[3]$} & 220.8331 & 64.6912 & 17.5308 & 0.9845 & \\
& {$[4]$} & 227.11 & 65.40 & 17.86 & 0.9132 & 8.976 \\
\hline \multirow{2}{*}{$10^{7}$} & this study & 702 & 148 & 39.5 & 1.34 & 16.5 \\
& {$[3]$} & 703.2536 & 145.2666 & 41.0247 & 1.3799 & \\
& {$[4]$} & 714.48 & 143.56 & 38.6 & 1.298 & 16.656 \\
\hline $10^{8}$ & this study & 2190 & 305 & 88.0 & 1.84 & 30.2 \\
& {$[3]$} & 2223.4424 & 283.689 & 91.2095 & 2.044 & \\
& {$[4]$} & 2259.08 & 296.71 & 91.16 & 1.766 & 31.486
\end{tabular}


Table 3

Comparison of the average Nusselt number as a function of the Rayleigh and Darcy numbers for the thermal convection in a fixed porous medium without advective and porosity effects $\left(k=1, c_{p}=1\right.$ and results are independent of $\left.\operatorname{Pr}\right)$.

\begin{tabular}{c|c|c|c|c}
$\overline{\mathrm{Da}}$ & $\mathrm{Ra}_{T}$ & this study & {$[5]$} & {$[6]$} \\
\hline $10^{-6}$ & $10^{7}$ & 1.08 & 1.07 & 1.08 \\
& $10^{8}$ & 3.08 & 3.06 & 3.004 \\
& $10^{9}$ & 13.2 & 13.22 & 12.25 \\
& $5 \times 10^{9}$ & 30.9 & 31.50 & \\
\hline $10^{-4}$ & $10^{5}$ & 1.07 & 1.06 & \\
& $10^{6}$ & 2.85 & 2.84 & \\
& $10^{7}$ & 10.3 & 10.34 & \\
& $5 \times 10^{7}$ & 20.1 & 20.85 & \\
\hline \multirow{2}{*}{$0^{-2}$} & $10^{3}$ & 1.02 & 1.02 & 1.023 \\
& $10^{4}$ & 1.71 & 1.70 & 1.708 \\
& $10^{5}$ & 4.26 & 4.26 & 4.26
\end{tabular}


Table 4

Comparison of the average Nusselt number as a function of the Rayleigh number, the Darcy number and the porosity for the thermal convection in a fixed porous medium with advective and porosity effects $\left(k=1, c_{p}=1\right.$ and $\left.\operatorname{Pr}=1\right)$. Corresponding values for both studies without advective and porosity effects can be found in table 3 . One should notice that Nithiarasu et al. [6] include in their computations an additional non-linear drag term, neglected in solidification simulations.

\begin{tabular}{|c|c|c|c|c|c|}
\hline$\overline{\mathrm{Da}}$ & $\mathrm{Ra}_{T}$ & $\begin{array}{l}\text { this study } \\
\epsilon_{0}=0.4\end{array}$ & $\begin{array}{c}{[6]} \\
\epsilon_{0}=0.4\end{array}$ & $\begin{array}{c}\text { this study } \\
\epsilon_{0}=0.9\end{array}$ & $\begin{array}{c}{[6]} \\
\epsilon_{0}=0.9\end{array}$ \\
\hline \multirow[t]{3}{*}{$10^{-6}$} & $10^{7}$ & 1.08 & 1.08 & 1.08 & 1.08 \\
\hline & $10^{8}$ & 3.07 & 2.99 & 3.08 & 3.01 \\
\hline & $10^{9}$ & 12.9 & 12.0 & 13.15 & 12.2 \\
\hline \multirow[t]{4}{*}{$10^{-2}$} & $10^{3}$ & 1.01 & 1.02 & 1.02 & 1.02 \\
\hline & $10^{4}$ & 1.41 & 1.69 & 1.67 & 1.70 \\
\hline & $10^{5}$ & 3.17 & 3.80 & 4.09 & 4.19 \\
\hline & $5 \times 10^{5}$ & 5.24 & 6.20 & 6.89 & 7.06 \\
\hline
\end{tabular}




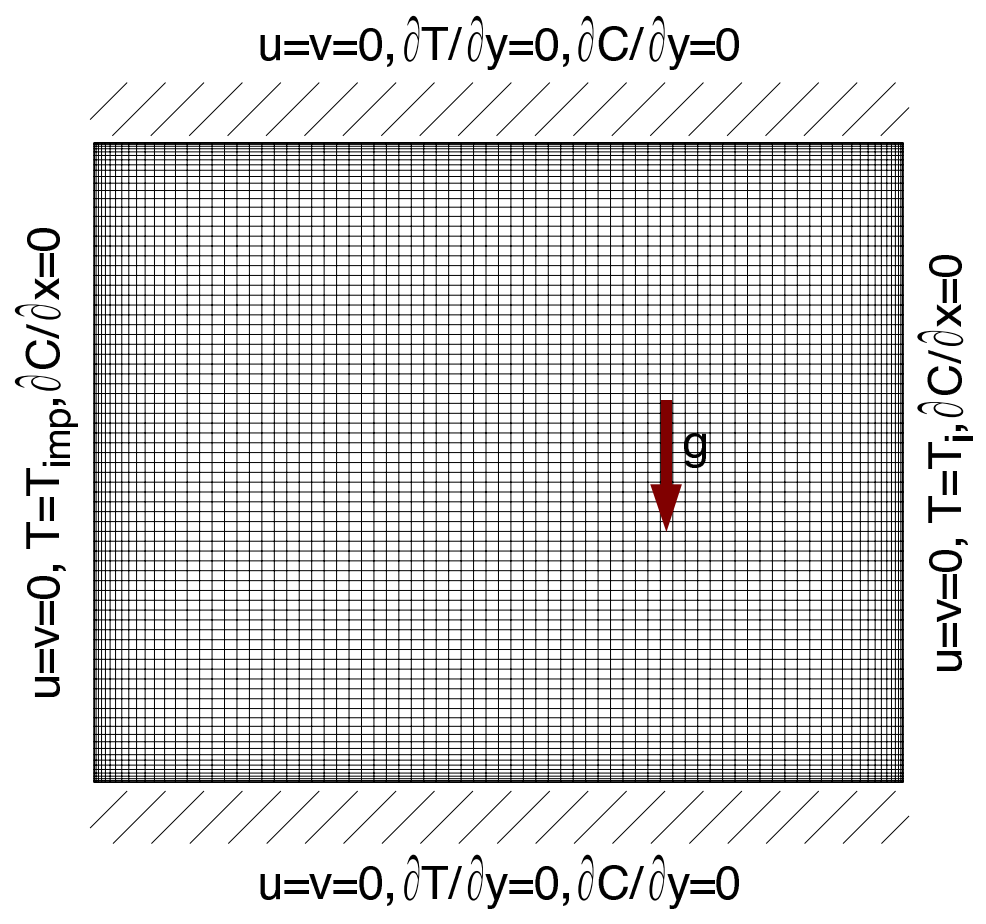

Fig. 1. Geometry, boundary conditions, and the $81 \times 81$ biased grid used in most computations. 


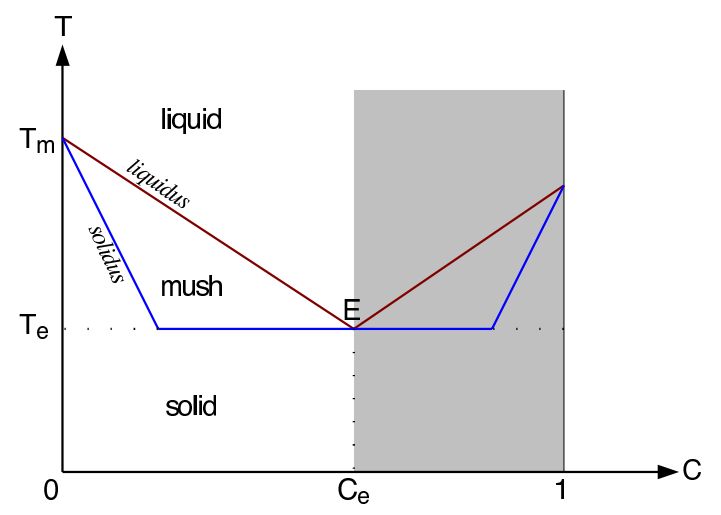

Fig. 2. Simplified phase diagram of a binary alloy. E is the eutectic point. 

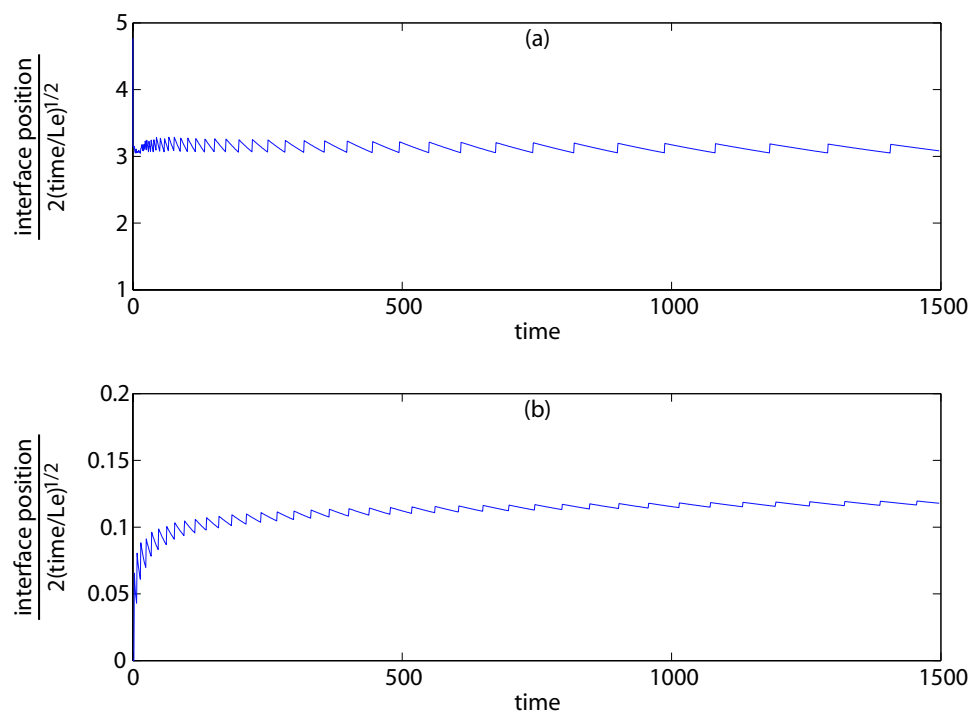

Fig. 3. Temporal evolution of the interface positions in diffusive solidification, normalised by the analytical law of Worster [1] for an imposed temperature $T_{i m p}=-18.6 K$ (i.e. $\left.T_{\text {liquidus }}\left(C_{i}\right)-T_{i m p}=13 K\right)$ : (a) mush-liquid interface and (b) mush-solid interface. Both ratios converge towards constants $\lambda_{a, b}$. The sawtooth shapes come from the discretisation in space: each sharp increase corresponds to a time at which the interface jumps from one grid point to the next one, whereas the continuous decreases between two jumps correspond to fixed interface position during time increase. These particular shapes imply an uncertainty of $\pm 4.5 \%$ on the determined values of the constants. For this computation, $\rho_{l}=\rho_{s}=1000$, $c_{p, l}=c_{p, s}=4.186 \times 10^{6} \mathrm{~J} \cdot \mathrm{m}^{-3} \cdot K^{-1}, k_{l}=k_{s}=0.5442 \mathrm{~W} \cdot \mathrm{m}^{-1} \cdot K^{-1}, p_{c}=0$, $C_{i}=0.14, C_{e}=0.80, T_{i}=15 K, T_{e}=-32 K, T_{m}=0 K, L=3.3488 \times 10^{8} J . m^{-3}$, $D=10^{-9} m^{2} \cdot s^{-1}$. 


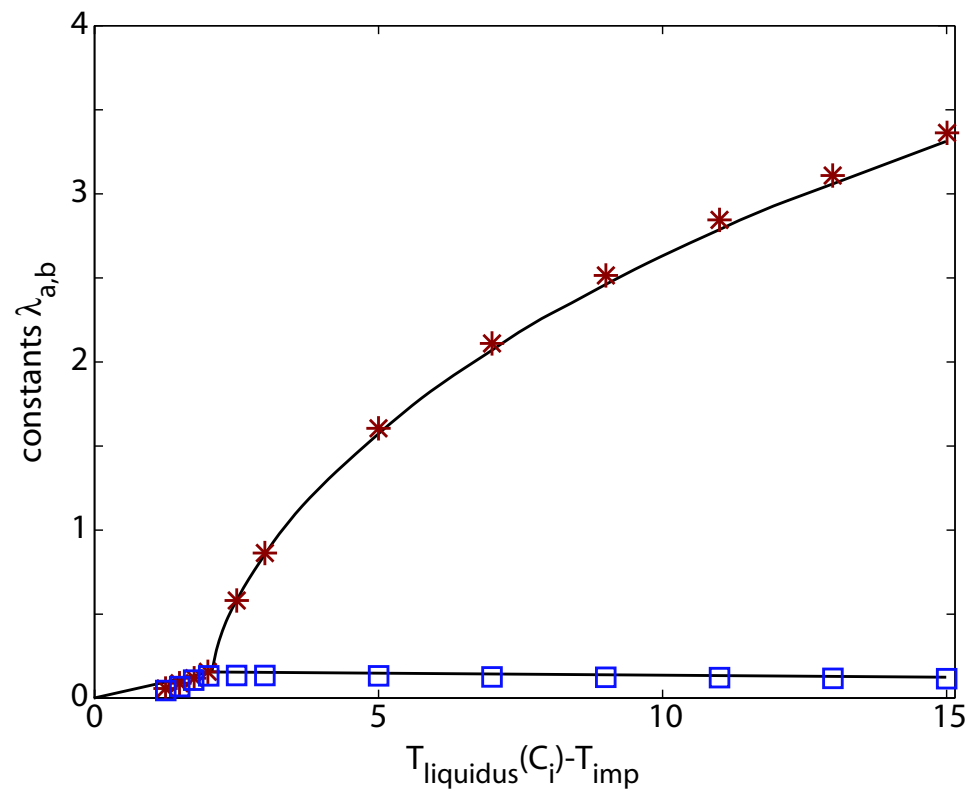

Fig. 4. Dimensionless constants $\lambda_{a, b}$ as a function of the temperature difference between the liquidus temperature at the initial concentration and the imposed temperature. A good agreement is found between analytical results of Worster [1] (continuous lines) and our numerical results (stars for the mush/liquid interface and squares for the mush/solid interface). Physical parameters are identical to figure 3. The size of the symbols includes the error bars due to the discretisation in space shown in figure 3. 
(a)

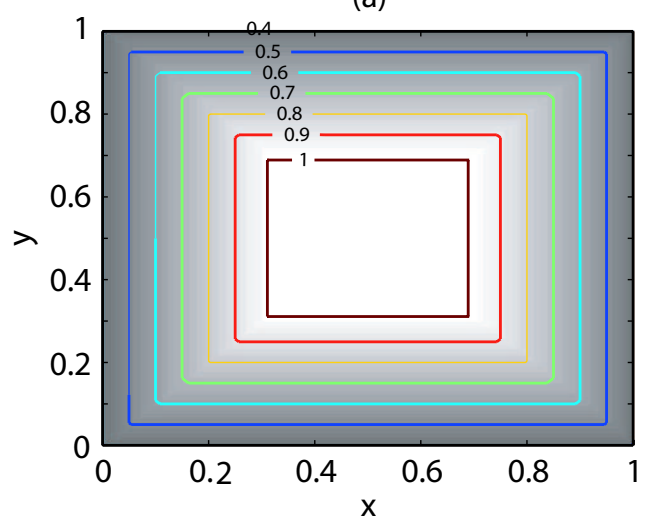

(c)

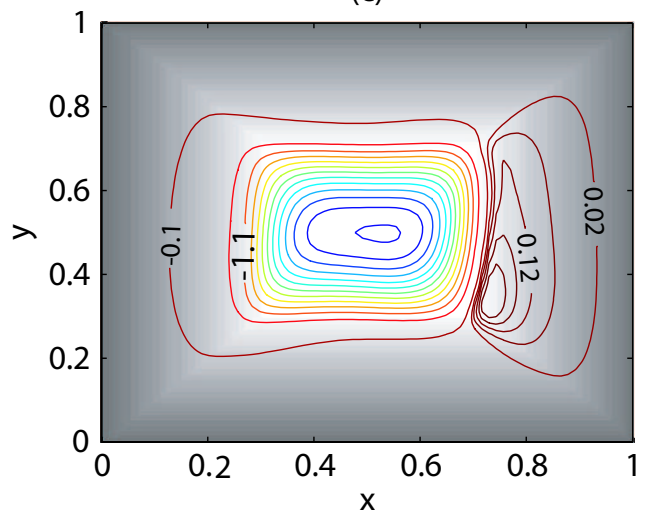

(b)

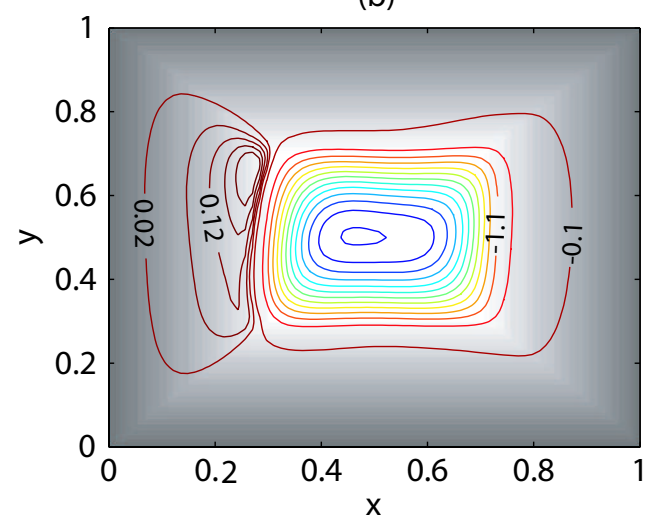

(d)

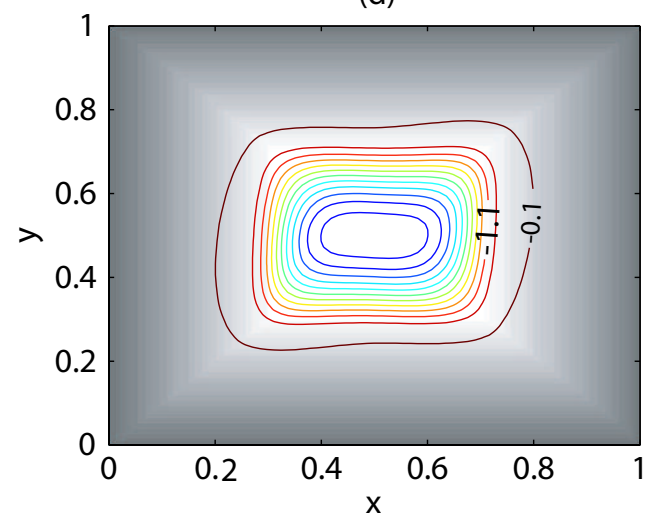

Fig. 5. Thermal convection in a fixed porous medium with a spatially-variable porosity (no solidification process takes place here). (a) Variations of the liquid fraction $\epsilon$, which changes linearly from 0.4 at the sides to 1 at a dimensionless distance greater than 0.3. ( $b, c$ and d) Computed streamlines in the case $R a_{T}=10^{6}, k=1, c_{p}=1$, $\operatorname{Pr}=1, D a=6.665 \times 10^{-7}$. Streamlines are equally spaced from -6.1 to -0.1 with a spacing 0.5 and from 0.02 to 0.22 with a spacing 0.05 . Imposed temperatures on the left/right sides are (b) $\theta=2.0 / 1.0$, (c) $\theta=-1.0 /-2.0$ and (d) $\theta=0.5 /-0.5$. 


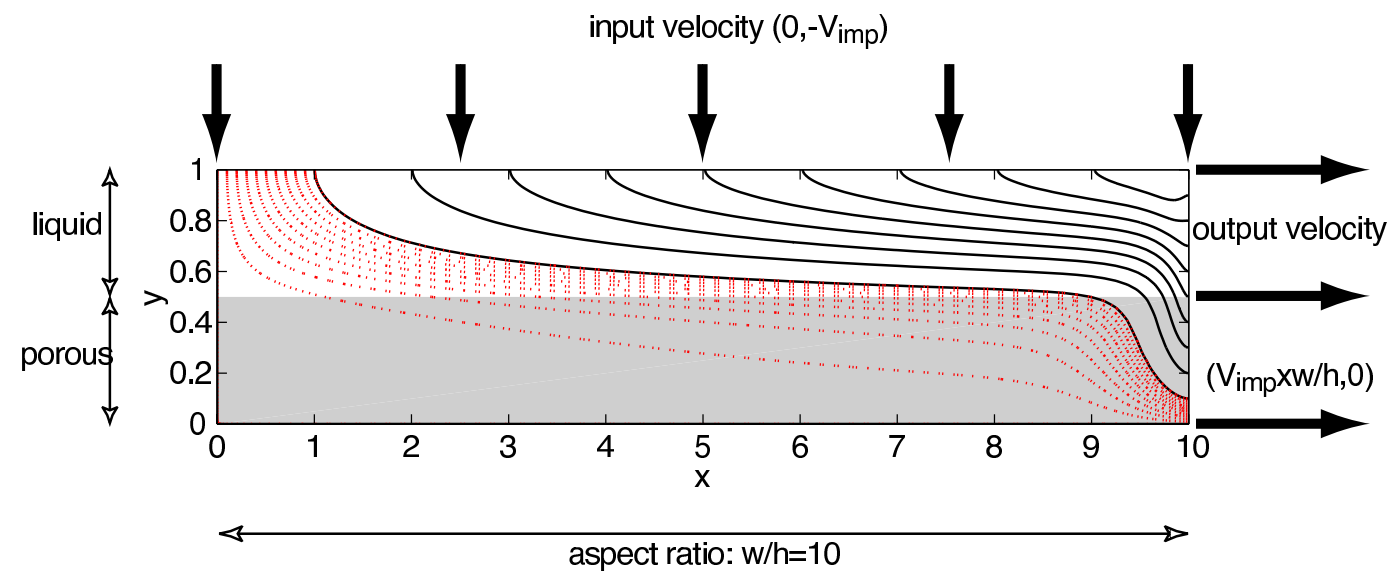

Fig. 6. Corner flow in a fluid overlying a porous layer with a constant porosity: geometry, boundary conditions and streamlines for $w / h=10, \epsilon_{0}=0.2, D a=0.08$, $V_{i m p}=10$. Continuous streamlines are equally spaced from 0 to 100 with a spacing 10 and dotted streamlines are equally spaced from 0 to 10 with a spacing 1. 

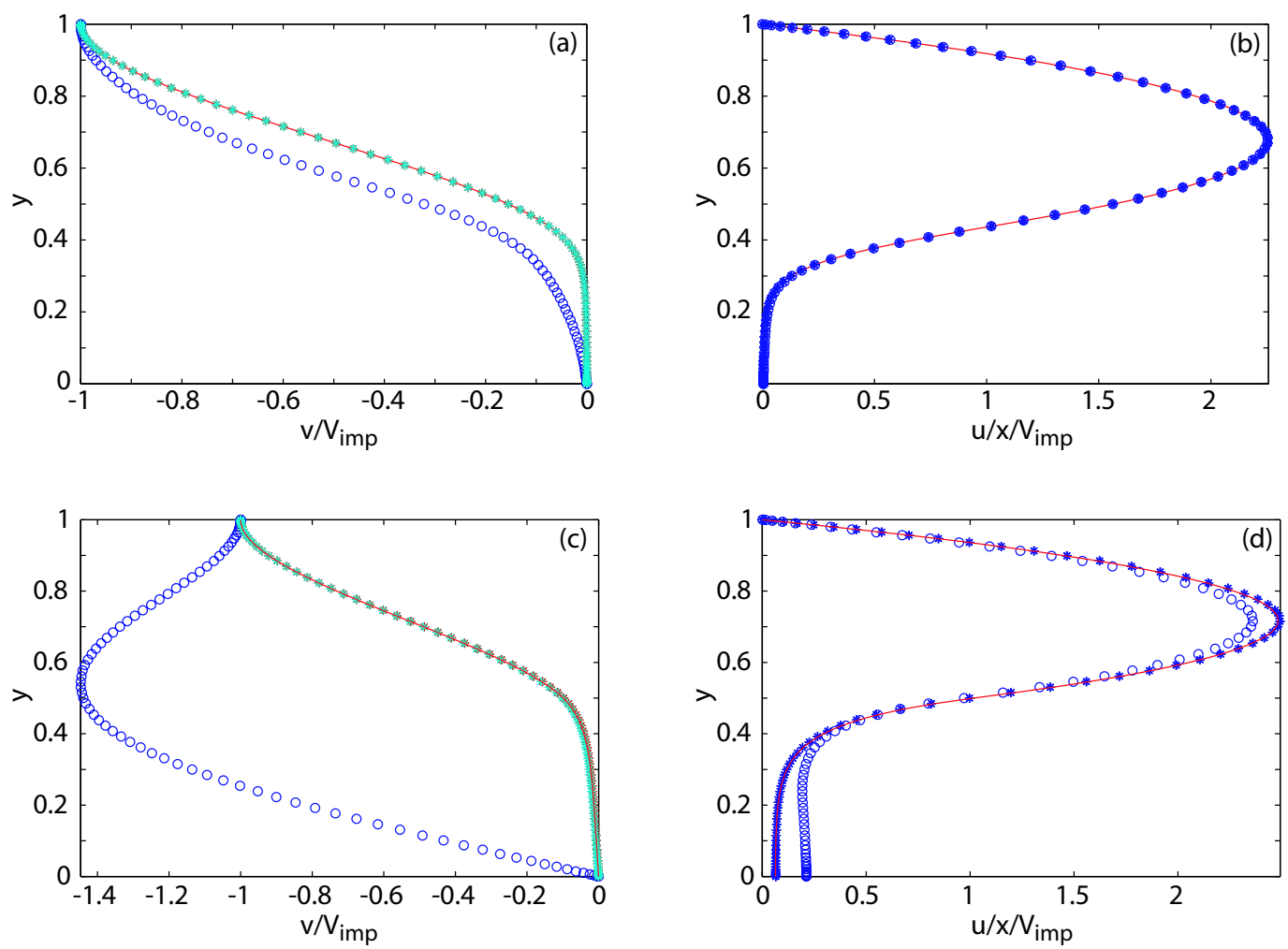

Fig. 7. (a,c) Vertical velocity and $(b, d)$ horizontal velocity divided by the horizontal position (see (31)) at various horizontal locations for a corner flow in a fluid overlying a porous layer with $(a, b)$ a linear porosity $\left(w / h=10, \epsilon_{0}=0.5\right.$ and $\left.D a=10^{-4}\right)$ and $(c, d)$ a constant porosity $\left(w / h=10, \epsilon_{0}=0.2\right.$ and $\left.D a=0.08\right)$. The continuous line is the similarity solution from Le Bars $\&$ Worster [8], stars represent the numerical solution measured at $x=0.01, x=2.5, x=5.0$, and $x=7.5$, whereas circles represent the numerical solution measured at $x=9.25$. The effects of boundary conditions are felt in the domain $9 \leq x \leq 10$ typically; all the other vertical profiles agree with the similarity solution. 

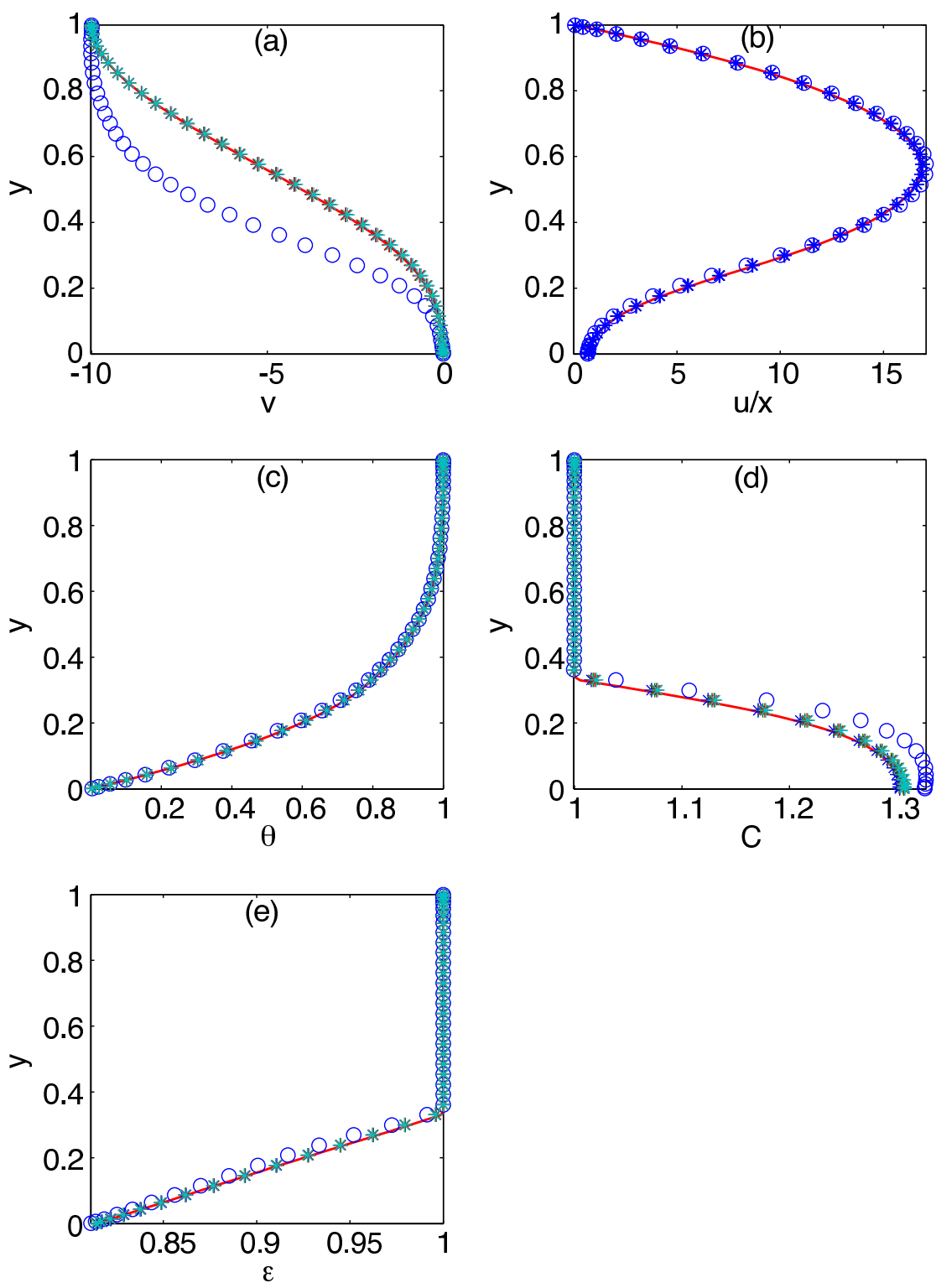

Fig. 8. Steady-state vertical profiles for the solidification of a binary alloy in a corner flow $\left(V_{\text {pull }}=1, V_{\text {imp }}=10, L=10, D a=10^{-4}, C_{e} /\left(C_{\infty}-C_{e}\right)=-7, m=0.8\right.$, $\left.L e=1000, p_{c}=0, c_{p}=1, k=1\right)$ : (a) vertical velocity, (b) horizontal velocity divided by the horizontal position (see (31)), (c) temperature, (d) bulk concentration and (e) liquid fraction. The continuous line is the similarity solution from Le Bars $\&$ Worster [8], stars represent the numerical solution measured at $x=0.01, x=2.5$, $x=5.0$, and $x=7.5$, whereas circles represent the numerical solution measured at $x=9.25$. The effect of boundary conditions is only felt in the domain $9<x<10$ typically. 